\title{
Kernel Fusion of Multiple Histogram Descriptors for Robust Face Recognition
}

\author{
Chi-Ho Chan, Josef Kittler, and Muhammad Atif Tahir \\ Centre for Vision, Speech and Signal Processing, \\ University of Surrey, United Kingdom \\ $\{$ c.chan, j.kittler,m.tahir\}@surrey.ac.uk
}

\begin{abstract}
A multiple kernel fusion method combining two multiresolution histogram face descriptors is proposed to create a powerful representation method for face recognition. The multi resolution histogram descriptors are based on local binary patterns and local phase coding to achieve invariance to various types of image degradation. The multikernel fusion is based on the computationally efficient spectral regression KDA. The proposed face recognition method is evaluated on FRGC 2.0 database yielding very impressive results.
\end{abstract}

Keywords: Local Binary Pattern, Local Phase Quantization, Kernel, Fusion, Linear Discriminant Analysis.

\section{Introduction}

Recognising faces under uncontrolled lighting conditions and blur either due to misfocus or motion is one of the most important challenges for practical face recognition systems. The problem is aggravated by a high dimensionality of the face data and a small sample size.

Most previous works on face recognition make use of raw image data as input to a linear transformation which maps the image to a point in a space, called face subspace. This point is defined by the coefficients of the face image projection into the associated bases, exemplified by [1] 2], Eigenface [3] and Fisherface [4]. However, the performance of such methods degrades when the cropped face image is acquired in changing illumination or is degraded by blur.

In contrast, histogram-based features, such as the Local binary pattern histogram (LBPH) [5], Local Phase Quantisation histogram (LPQH) 6] and the histogram of Gabor Phase Patterns (HGPP) 7], have gained reputation as powerful and attractive texture descriptors showing excellent results in terms of accuracy and computational complexity in face recognition, as these features, which capture the information about the spatial relation of facial regions, are partially invariant to these degradation. In these methods, the face image is first partitioned into a large number of small regions from which pattern histograms, representing the local texture of face images, are extracted. The recognition is performed using the nearest-neighbour classifier. Chan et al. have extended the LBP histogram [8] and LPQ histogram 9] methods to provide a multiresolution

E.R. Hancock et al. (Eds.): SSPR \& SPR 2010, LNCS 6218, pp. $718-727,2010$.
(C) Springer-Verlag Berlin Heidelberg 2010 
representation which further exhibits robustness to face misalignment. These extensions have been demonstrated to achieve excellent results in Feret, XM2VTS and BANCA databases. It is well known that multiple cues enrich the representation of any object. This has been demonstrated also for faces. Face representations derived from the complementary sources of information presented in Table 1 have been shown to achieve better performance than single best representation. It is evident from Table 1 that with the exception of 10 the reviewed fusion studies always involve integrating information emanating from different image domains rather than using different face representations which are derived from a single domain. Moreover, most systems in Table 1 apply either a score fusion or a feature level fusion method. It is therefore of interest to investigate a novel mechanism -multiple kernel fusion- for combining different face representations computed from an intensity image. We focus on the an intensity domain, as it is more robust to changes in image acquisition conditions (camera, illumination).

Table 1. Summary of the fusion methods in different face recognition systems

\begin{tabular}{|c|c|c|c|}
\hline Ref. & Image Domain & Face representation & Fusion Method \\
\hline $\mathbf{1 1}$ & Infrared and Visible Images & Wavelet & Feature fusion \\
\hline $\mathbf{1 2}$ & $2.5 \mathrm{D}$, Curvature, Visible Images & Gabor & Kernel fusion \\
\hline $\mathbf{1 3}$ & Color Image & LBPH & Feature and Score fusions \\
\hline $\mathbf{1 0}$ & Intensity Image & Gabor, LBPH & Score fusion \\
\hline $\mathbf{1 4}$ & Color Image & Frequency feature & Score fusion \\
\hline $\mathbf{1 5}$ & Color Image & Gabor, MLBPH, Frequency Feature & Score fusion \\
\hline $\mathbf{1 6}$ & Global, intrinsic faces & Frequency Feature & Score fusion \\
\hline $\mathbf{1 7}$ & Global, intrinsic faces & Gabor, Frequency Feature & Score fusion \\
\hline
\end{tabular}

This paper presents a computational and statistical framework for integrating two different descriptors, Multiscale LBPH and Multiscale LPQH for face recognition in 2D grey-scale image domain. These descriptors are selected because of their invariance to monotonic illumination changes and blur. The framework relies on the use of kernel-based statistical learning methods. These methods represent the data by means of a kernel function which is the non-linear function of similarities between pairs of face descriptors. One of the reason for the success of kernel methods is that the kernel function measures the similarity between query face image descriptors and those derived from the training set in an implicitly infinitely dimensional space. Each kernel therefore extracts a specific type of information from the training set, thus providing a partial description or view of the query image. A unique combined kernel obtained from the individual kernels formed by the two descriptors is then projected into the Fisher space for face recognition. Paper is organised as follows. In Section 2 we introduce the image descriptors adopted, as well as a computationally efficient kernel matching method. The problem of fusion is discussed in Section 3. The experimental results are presented in Section 4, leading to conclusions in Section 5 . 


\section{Histogram Features}

\section{Local Binary Pattern}

The LBP operator, shown in Equation 1, extracts information which is invariant to local monotonic grey-scale variations of the image. During the LBP operation, the value of current pixel, $f_{c}$, is applied as a threshold to each of the neighbours, $f_{p}(p=0, \cdots, P-1)$ to obtain a binary number. A local binary pattern is obtained by first concatenating these binary bits and then converting the sequence into the decimal number. Using circular neighbourhoods and linearly interpolating the pixel values allows the choice of any radius, $R$, and number of pixels in the neighbourhood, $\mathrm{P}$, to form an operator

$$
L B P_{P, R}(\mathbf{x})=\sum_{p=0}^{P-1} s\left(f_{p}-f_{c}\right) 2^{P} \quad \mid \quad s(v)= \begin{cases}1 & v \geq 0 \\ 0 & v<0\end{cases}
$$

\section{Local Phase Quantisation Pattern}

The local phase quantisation(LPQ) [6 pattern is robust to blur effects. The phase information of LPQ can be extracted using the two dimensional windowed Fourier transform (2DWFT).

$$
\mathbf{F}_{\mathbf{u}}(\mathbf{x})=\sum_{\mathbf{m} \in \mathcal{N}_{x}} \mathbf{w}(\mathbf{m}-\mathbf{x}) \mathbf{f}(\mathbf{m}) e^{-j 2 \pi \mathbf{u}^{T} \mathbf{m}}=\mathbf{E}_{u}^{T} \mathbf{f}_{\mathbf{x}}
$$

where $\mathbf{E}_{u}$, size $=1 \times z^{2}$, is a basis vector of 2 DWFT with frequency $\mathbf{u}$, and $\mathbf{f}_{\mathbf{x}}$, size $=z^{2} \times N$, is a vector containing image pixel values in $\mathcal{N}_{x}$ at each $\mathbf{x}$ location. The window function, $\mathbf{w}(\mathbf{x})$ is a rectangular function in this work. The transform is computed at four frequency points, $\mathbf{u}=\left[\mathbf{u}_{0}, \mathbf{u}_{1}, \mathbf{u}_{2}, \mathbf{u}_{3}\right]$ where $\mathbf{u}_{0}=[a, 0]^{T}$, $\mathbf{u}_{1}=[0, a]^{T}, \mathbf{u}_{2}=[a, a]^{T}$ and $\mathbf{u}_{3}=[a,-a]^{T} . a$ is a highest scalar frequency for which $\mathbf{W}_{\mathbf{u}_{i}}>0$. Thus, only four exponential complex functions are needed as a filter bank to yield eight resultant complex images consisting of 4 filtered images of the real part and 4 images of the imaginary part of the transform. A whitening transform [6] is applied to decorrelate $\mathbf{F}_{\mathbf{u}}(\mathbf{x})$ to improve the system performance. Each pixel of the resultant complex image can be encoded into a binary value shown in Equation (3) by applying the quadrant bit coding.

$$
\begin{aligned}
& \mathbf{B}_{\mathbf{u}_{i}}^{R e}(\mathbf{x})= \begin{cases}1 & \text { if } \mathbf{F}_{\mathbf{u}_{i}}^{R e}(\mathbf{x})>0 \\
0 & \text { if } \mathbf{F}_{\mathbf{u}_{i}}^{R e}(\mathbf{x}) \leq 0\end{cases} \\
& \mathbf{B}_{\mathbf{u}_{i}}^{I m}(\mathbf{x})= \begin{cases}1 & \text { if } \mathbf{F}_{\mathbf{u}_{i}}^{I m}(\mathbf{x})>0 \\
0 & \text { if } \mathbf{F}_{\mathbf{u}_{i}}^{I m}(\mathbf{x}) \leq 0\end{cases}
\end{aligned}
$$

This coding method assigns 2 bits for every pixel to represent the quadrant in which the phase angle lies. In fact, it also provides the quantisation of the Fourier phase feature. LPQ is a binary string obtained, for each pixel, by concatenating 
the real and imaginary quadrant-bit codes of the eight Fourier coefficients of $\mathbf{u}_{i}$. The binary string is then converted to the decimal number by Equation (4) to produce a LPQ pattern

$$
\begin{array}{r}
\mathbf{L P Q}(\mathbf{x})=\mathbf{B}_{\mathbf{u}_{0}}^{R e}(\mathbf{x})+\mathbf{B}_{\mathbf{u}_{0}}^{I m}(\mathbf{x}) \times 2^{1}+\cdots \\
+\mathbf{B}_{\mathbf{u}_{3}}^{R e}(\mathbf{x}) \times 2^{k-1}+\mathbf{B}_{\mathbf{u}_{3}}^{I m}(\mathbf{x}) \times 2^{k}
\end{array}
$$

In digital image processing, blur effects can be modelled by a discrete linear relationship defined by a convolution between the image intensity and a point spread function (PSF). In the Fourier transform, the phase of each harmonic of the blurred image is the sum of the phase of the original image and phase of the PSF. If the PSF of blur is a positive even function, it will act as a zero-phase low-pass filter. In other words, the LPQ representation is invariant to blur if the cut-off frequency of blur (PSF) is greater than that of the LPQ filter.

\section{Multiscale Pattern Histogram}

A multiresolution representation can be obtained by varying the filter size, $z \times z$, and combining the resulting pattern images. Such a representation [9] [8] has been suggested for face recognition and the results reported for this application show that the accuracy is better than that of a single scale pattern method. As a multiresolution representation defined by a set of pattern operators of different filter size may give an unstable result because of noise, this problem can be minimised by using aggregate statistics, exemplified by histogram. There are several advantages in summarising the patterns in the form of histogram. First, the statistical summary can reduce the feature dimension from the image size to the number of histogram bins. Secondly, using histogram as a set of features is robust to image translation and rotation to a certain extent and therefore the sensitivity to mis-registration is reduced. Finally, although the effect of unstable pattern responses due to noise is attenuated by histogramming, it can further be reduced by controlling the number of histogram bins and /or projecting the histogram to other spaces.

In our approach, pattern operators defined on a neighbourhood $Q$, for an instance LBP or LPQ, at R scales, are first applied to a face image. This generates a grey level code for each pixel at every resolution. The resulting Pattern images are cropped to the same size and divided into non-overlapping sub-regions, $\mathbf{M}_{0}$, $\mathbf{M}_{1}, . . \mathbf{M}_{J-1}$. The regional pattern histogram for each scale is computed as

$$
\begin{gathered}
\mathbf{h}_{r, j}(i)=\sum_{\mathbf{x} \in \mathbf{M}_{j}} E\left(Q_{r}(\mathbf{x})=i\right) \\
\mid i \in[0, L-1], j \in[0, J-1], \\
r \in[1, R], z=r \times 2+1
\end{gathered}
$$


$E(v)$ is a Boolean indicator. $r$ is the scale index and $z$ is the width or height of the pattern filter. The set of histograms computed at different scales for each region $\mathbf{M}_{j}$ provides regional information. $L$ is the number of histogram bins. By concatenating these histograms into a single vector, we obtain the final multiresolution regional face descriptor.

$$
\mathbf{k}_{j}=\left[\mathbf{h}_{1, j}, \mathbf{h}_{2, j}, \cdots, \mathbf{h}_{R, j}\right]
$$

\section{KDA Using Spectral Regression (SR-KDA)}

Kernel Discriminant Analysis is a non-linear extension of LDA which maps the original measurements into a higher dimensional space using the "kernel trick". If $\nu$ denotes a projective function into the kernel feature space, then the objective function for KDA is

$$
\max _{\nu} \mathcal{J}(\nu)=\frac{\nu^{T} \mathbf{C}_{b} \nu}{\nu^{T} \mathbf{C}_{t} \nu}
$$

where $\mathbf{C}_{b}$ and $\mathbf{C}_{t}$ denote the between-class and total scatter matrices in the feature space respectively. A solution to Equation 7 leads to the eigenvalue analysis problem $\mathbf{C}_{b}=\lambda \mathbf{C}_{t}$. It is proved in [18] that equation $\mathbf{7}$ is equivalent to

$$
\max _{\alpha} \mathcal{J}(\mathbf{w})=\frac{\mathbf{w}^{T} \mathbf{K A K w}}{\mathbf{w}^{T} \mathbf{K K} \mathbf{w}}
$$

where $\mathbf{w}=\left[\alpha_{1}, \alpha_{2}, \cdots, \alpha_{m}\right]^{T}$ is the eigen-vector satisfying $\mathbf{K A K} \mathbf{w}=\lambda \mathbf{K K} \mathbf{w}$. $\mathbf{A}=\left(\mathbf{A}_{l}\right)_{l=1, \cdots, n}$ is a $(m \times m)$ block diagonal matrix of labels arranged such that the upper block corresponds to positive examples and the lower one to negative examples of the class. $\mathbf{K}$ is an $m \times m$ kernel matrix such that $K\left(\mathbf{k}^{s_{1}}, \mathbf{k}^{s_{2}}\right)=$ $\left\langle\Phi\left(\mathbf{k}^{s_{1}}\right), \Phi\left(\mathbf{k}^{s_{2}}\right)\right\rangle$, where $\Phi\left(\mathbf{k}^{s_{1}}\right)$ and $\Phi\left(\mathbf{k}^{s_{2}}\right)$ are the embeddings of data items $\mathbf{k}^{s_{1}}$ and $\mathbf{k}^{s_{2}}$. Each eigenvector $\mathbf{w}$ gives a projection function $\nu$ into the feature space.

It is shown in [19 that instead of solving the eigen-problem in KDA, the KDA projections can be obtained by the following two linear equations

$$
\begin{aligned}
\mathbf{A} \phi & =\lambda \phi \\
(\mathbf{K}+\delta \mathbf{I}) \mathbf{w} & =\phi
\end{aligned}
$$

where $\phi$ is an eigenvector of $\mathbf{A}, \mathbf{I}$ is the identity matrix and $\delta>0$ is a regularisation parameter. Eigen-vectors $\phi$ are obtained directly from the GramSchmidt method. Since $(\mathbf{K}+\delta \mathbf{I})$ is positive definite, the Cholesky decomposition, $(\mathbf{K}+\delta \mathbf{I})=\mathbf{R}^{T} \mathbf{R}$ is used to solve the linear equations in Equation 9 and the obtained result, $R$ is a upper triangular matrix. Thus, the solution of the linear system becomes

$$
(\mathbf{K}+\delta \mathbf{I}) \mathbf{w}=\phi \Leftrightarrow\left\{\begin{array}{l}
\mathbf{R}^{T} \theta=\phi \\
\mathbf{R} \mathbf{w}=\theta
\end{array}\right.
$$

i.e., first solve the system to find vector $\theta$ and then vector w. In summary, SRKDA, $\mathbf{W}^{k d a}=\left[\mathbf{w}_{1}, \mathbf{w}_{2}, \cdots, \mathbf{w}_{c-1}\right]$, only needs to solve a set of regularised regression problems and there is no eigenvector computation involved. This results 
in great improvement of computational cost and allows to handle large kernel matrices.

Complexity Analysis. The computation of SR-KDA involves two steps: (i) response generation which is the cost of the Gram-Schmidt method, and (ii) regularised regression which involves solving $(c-1)$ linear equations using the Cholesky decomposition where $c$ is the number of classes. As in 20, we use the term flam, a compound operation consisting of one addition and one multiplication, to measure the operation counts. The cost of the Gram-Schmidt method requires $\left(m c^{2}-\frac{1}{3} c^{3}\right)$ flams. The Cholesky decomposition requires $\frac{1}{6} m^{3}$ flams and the $c-1$ linear equations can be solved with $m^{2} c$ flams. Thus, the computational cost of SRKDA excluding the cost of Kernel Matrix $\mathbf{K}$ is $\frac{1}{6} m^{3}+m^{2} c+m c^{2}-\frac{1}{3} c^{3}$ which can be approximated as $\frac{1}{6} m^{3}+m^{2} c$. Comparing to the cost of ordinary KDA $\left(\frac{9}{2} m^{3}+m^{2} c\right)$, SR-KDA significantly reduces the dominant part and achieves an order of magnitude (27 times) speed-up.

\section{System Fusion}

We investigate two frameworks for information fusion: Score level fusion and Kernel level fusion as shown in Figure 1

Score level fusion: In the case of score-level fusion, for each representation the face recognition system is trained individually using SR-KDA. The output from each classifier is then combined using the sum rule.

Kernel level fusion: Given multiple features (MLPQ, MLBP), each kernel function produces a square matrix in which each entry encodes a particular notion of similarity of one face to another. This kernel formalism also allows these multiple features to be combined. Basic algebraic operations such as addition maintain the key property of positive semi-definiteness and thus allow a simple but powerful algebra of kernels. For example, it is possible to combine kernels computed from MLPQ and MLBP such that kernel $\mathbf{K}=\mathbf{K}_{M L P Q H}+\mathbf{K}_{M L B P H}$. Once the kernels are combined, SR-KDA is then applied for feature extraction. It should be noted that this Kernel-level fusion has a speed advantage over the score-level fusion as only one classifier is required. In contrast, for score-level fusion, separate classifiers are required for the individual face representations.

\section{Experimental Result}

The Face Recognition Grand Challenge version 2 data set is used to evaluate the proposed framework. The faces of this database collected in controlled and uncontrolled environments are divided into training and test sets. The training set contains 12,775 images from 222 subjects, while the test set data contains 24,042 images from 466 in which 222 subjects are common to the training set but their image are not shared with the training set. We focus on experiments, EXP 1 and 4, in this work. EXP 1 is designed to measure the performance 


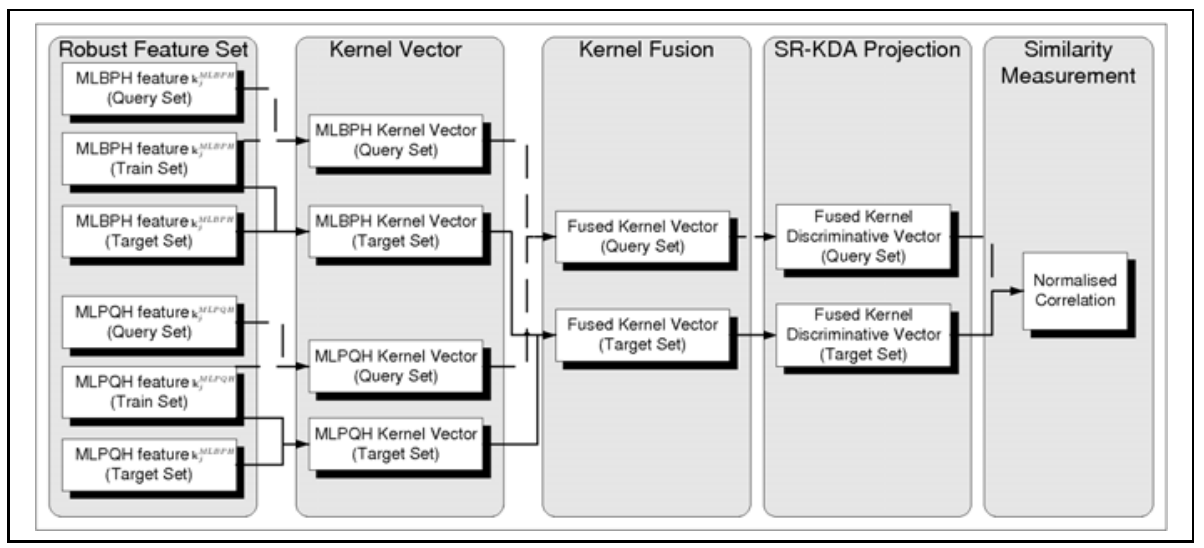

(a) Kernel

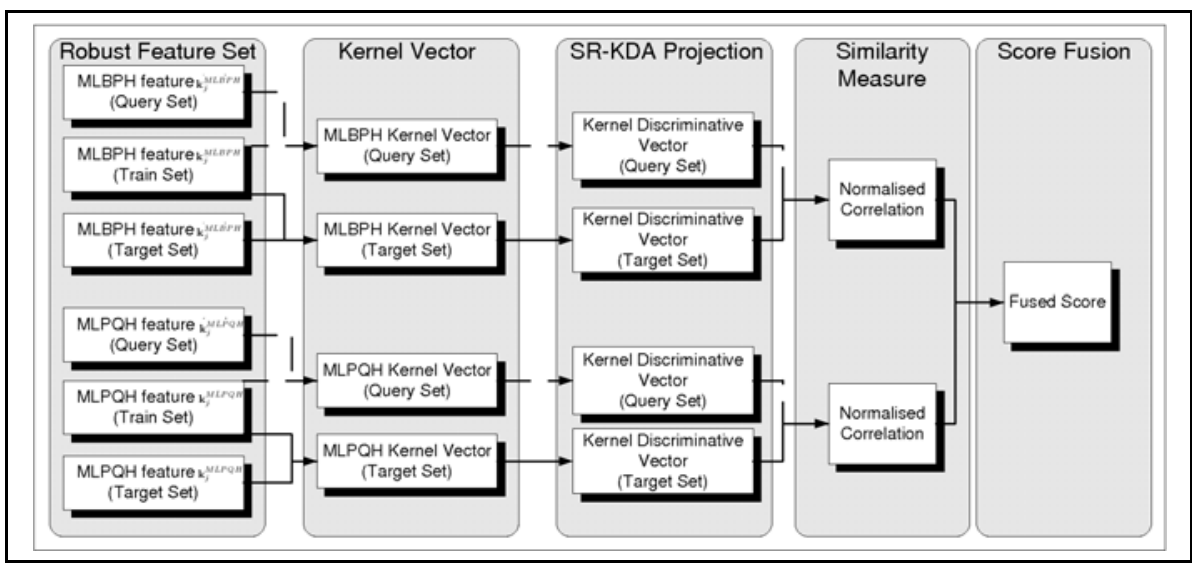

(b) Score

Fig. 1. Block diagrams of Kernel fusion and score fusion methods

of face verification from frontal images taken under controlled illumination. In EXP 1, 16,028 images from 466 subjects are used to establish 16,028 $\times 16,028$ similarity confusion matrix. EXP 4 is designed to measure the performance on controlled versus uncontrolled frontal face still images. The target set consists of 16,028 controlled images and the query set contains 8,014 uncontrolled still images. The ROC curve plotting the Face Verification rate (FVR) versus the False Acceptance Rate (FAR) is generated using the Biometric Experimentation Environment (BEE) evaluation tool. It produces three ROC curves (ROC I, II \& III) corresponding to the images acquired within semesters, within a year, and between semesters, respectively.

Face images are extracted with the ground-truth annotated eye positions and scaled to a size of $142 \times 120$ (rows $\times$ columns). The cropped faces are photometrically normalised by the Preprocessing sequence approach(PS) [10]. This 
Table 2. The verification rate in $\%$ at $0.1 \%$ FAR for different methods on FRGC 2.0 Experiment 1 and 5

\begin{tabular}{|l|c|c|c||c|c|c|}
\hline System & \multicolumn{3}{|c|}{ EXP 1} & \multicolumn{3}{c|}{ EXP 4} \\
\hline & ROC & ROC II & ROC III & ROC I & ROC II & ROC III \\
\hline PS_MLBP+LDA & 97.11 & 96.31 & 95.40 & 67.32 & 68.92 & 70.51 \\
\hline PS_MLBP+KDA(ED) & 98.37 & 97.91 & 97.42 & $\mathbf{7 7 . 8 5}$ & $\mathbf{7 9 . 6 8}$ & $\mathbf{8 1 . 5 3}$ \\
\hline PS_MLBP+KDA(X2) & $\mathbf{9 8 . 4 8}$ & $\mathbf{9 8 . 0 3}$ & $\mathbf{9 7 . 5 5}$ & 75.45 & 77.67 & 79.81 \\
\hline \hline PS_MLPQ+LDA & 97.30 & 96.51 & 95.67 & 67.32 & 68.86 & 70.50 \\
\hline PS_MLPQ+KDA(ED) & $\mathbf{9 8 . 7 6}$ & $\mathbf{9 8 . 3 9}$ & $\mathbf{9 7 . 9 8}$ & $\mathbf{8 1 . 0 5}$ & $\mathbf{8 2 . 4 4}$ & $\mathbf{8 3 . 8 0}$ \\
\hline PS_MLPQ+KDA(X2) & $\mathbf{9 8 . 7 6}$ & 98.37 & 97.94 & 78.27 & 79.98 & 81.54 \\
\hline \hline PS_MLPQ+LDA+PS_MLBP+LDA & 97.45 & 96.69 & 95.89 & 69.73 & 71.59 & 73.43 \\
\hline PS_MLPQ+KDA(ED)+PS_MLBP+KDA(ED) & 98.70 & 98.31 & 97.88 & $\mathbf{8 1 . 1 7}$ & $\mathbf{8 2 . 8 4}$ & $\mathbf{8 4 . 4 2}$ \\
\hline PS_MLPQ+KDA(X2)+PS_MLBP+KDA(X2) & $\mathbf{9 8 . 7 7}$ & $\mathbf{9 8 . 3 8}$ & $\mathbf{9 7 . 9 5}$ & 79.15 & 81.04 & 82.87 \\
\hline \hline PS_MLPQ+PS_MLBP+KDA(ED) & 98.84 & 98.50 & 98.13 & $\mathbf{8 2 . 9 2}$ & $\mathbf{8 4 . 3 4}$ & $\mathbf{8 5 . 7 2}$ \\
\hline PS_MLPQ+PS_MLBP+KDA(X2) & $\mathbf{9 8 . 8 8}$ & $\mathbf{9 8 . 5 4}$ & $\mathbf{9 8 . 1 6}$ & 80.50 & 82.19 & 83.78 \\
\hline \hline CVPR06'[16] & 95.01 & 93.29 & 91.51 & 75.70 & 75.06 & 74.33 \\
\hline ICCV07'[17 & & & 98.00 & & & 86.00 \\
\hline AMFG07'[22] & & & & & & 83.60 \\
\hline INNS09'[13] & & & & & & 83.4 \\
\hline TIP08' 14] & & & & 79.40 & 79.90 & 80.30 \\
\hline ICB09' 15] & & & & & & 92.40 \\
\hline CVPR05'LBP+KLDA(X2) 23] & 97.40 & & & & & \\
\hline CVPR05'LBP [23] & 79.90 & & & & & \\
\hline CVPR05'KLDA [23] & 82.90 & & & & & \\
\hline PCA_Baseline & & & 74.76 & 70.53 & 66.05 & 12.00 \\
\hline
\end{tabular}

photometric normalisation method is designed to reduce the effects of illumination variation, local shadowing and highlights, while still keeping the essential visual appearance information for the use in recognition. Our objective is to evaluate the MLPQH and MLBPH descriptors and their combination. For MLBPH, ten LBP operators from $r=1$ to 10 with $P=8$ are employed to represent the face image, while eight LPQ operators from $z=3$ to 17 for MLPQ. The coded images are then divided into 9 non-overlapping regions and the kernel vectors based on the local histograms are generated in the testing stage. In this work, we have used RBF kernel with Chi-squared (X2) and Euclidean distance (ED) metrics: $K\left(\boldsymbol{k}^{\boldsymbol{s 1}}, \boldsymbol{k}^{\boldsymbol{s} \mathbf{2}}\right)=e^{-\frac{1}{A} \operatorname{dist}\left(\boldsymbol{k}^{\boldsymbol{s 1}}, \boldsymbol{k}^{\boldsymbol{s} \mathbf{2}}\right)}$ where $A$ is a scalar which normalises the distances. Following [21], $A$ is set to the average Chi-squared or Euclidean distance between all elements of the kernel matrix. The default value of regularisation parameter $\delta=0.01$ is used in all experiments.

In kernel fusion (MLBPH+MLPQH_KDA), the kernel vectors of MLBPH and MLPQH in each region are fused together and projected into SRKDA space to represent the regional discriminative facial descriptors. The final similarity score is obtained by summing the similarity, i.e. normalized correlation, of regional discriminative descriptors. On the other hand, in Score level fusion (MLBPH_KDA+MLPQH_KDA), SRKDA is applied to each of histogram descriptors and then the similarity score is fused by averaging the similarity scores. For the benchmark systems, the score level fusion of LDA version of MLBPH and 
MLPQH (MLBPH_LDA+MLPQH_LDA), MLPQH_LDA and MLBPH_LDA are evaluated and the state of art methods are also reported in Table 2.

Compared to Linear Discriminant analysis-based systems, the Kernel Discriminant analysis performs significantly better in EXP 1 . However, there is no significant difference between the performance of the RBF kernel with Chi-squared (X2) and Euclidean distance (ED) metrics. As expected, the performance obtained when combining two different face representations is better than the performance of the individual representation, except for PS_MLPQ+KDA(ED) + PS_MLBP $+\mathrm{KDA}(\mathrm{ED})$ in EXP 1. Kernel fusion always outperforms score level fusion. Our proposed frameworks using kernel fusion to combine two different face representations achieves slightly better performance than the system combining the scores from global and intrinsic face images [17. However, the result of our proposed method is not better than the method in ICB2009 [15] where this complicated method integrating different face representations, such as LBP, Gabor and Fourier features in colour domain achieves better performance. Nevertheless, we argue that any system using colour may not be robust in the real environment and also has a heavy computational cost.

\section{Conclusions}

We have presented a kernel fusion method for integrating two new robust descriptors for face recognition under uncontrolled lighting conditions and blur. Tested on the challenging FRGC 2.0 database, our proposed framework achieves better performance than the score level fusion. It also outperforms all state of the art method in comparable conditions. The proposed method provides an alternative solution for integrating the descriptors together to achieve robust performance.

Acknowledgements. This work is supported by the EU-funded Mobio project grant IST-214324,(www.mobioproject.org).

\section{References}

1. Wiskott, L., Fellous, J.M., Krüger, N., von der Malsburg, C.: Face recognition by elastic bunch graph matching. PAMI 19(7), 775-779 (1997)

2. Zhang, W., Shan, S., Gao, W., Chen, X., Zhang, H.: Local gabor binary pattern histogram sequence (lgbphs): A novel non-statistical model for face representation and recognition. In: ICCV, pp. 786-791 (2005)

3. Turk, M.A., Pentland, A.: Face recognition using eigenfaces. In: CVPR, pp. 586-591 (1991)

4. Belhumeur, P., Hespanha, J., Kriegman, D.: Eigenfaces vs. fisherfaces: recognition using class specific linear projection. PAMI 19(7), 711-720 (1997)

5. Ahonen, T., Hadid, A., Pietikäinen, M.: Face recognition with local binary patterns. In: Pajdla, T., Matas, J(G.) (eds.) ECCV 2004. LNCS, vol. 3021, pp. 469-481. Springer, Heidelberg (2004)

6. Ahonen, T., Rahtu, E., Ojansivu, V., Heikkilä, J.: Recognition of blurred faces using local phase quantization. In: ICPR, pp. 1-4 (2008) 
7. Zhang, B., Shan, S., Chen, X., Gao, W.: Histogram of gabor phase patterns (hgpp): A novel object representation approach for face recognition. IEEE Transactions on Image Processing 16(1), 57-68 (2007)

8. Chan, C., Kittler, J., Messer, K.: Multi-scale local binary pattern histograms for face recognition. In: Lee, S.-W., Li, S.Z. (eds.) ICB 2007. LNCS, vol. 4642, pp. 809-818. Springer, Heidelberg (2007)

9. Chan, C., Kittler, J., Poh, N., Ahonen, T., Pietikäinen, M. (multiscale) local phase quantization histogram discriminant analysis with score normalisation for robust face recognition. In: VOEC, pp. 633-640 (2009)

10. Tan, X., Triggs, B.: Enhanced local texture feature sets for face recognition under difficult lighting conditions. In: Zhou, S.K., Zhao, W., Tang, X., Gong, S. (eds.) AMFG 2007. LNCS, vol. 4778, pp. 168-182. Springer, Heidelberg (2007)

11. Singh, S., Gyaourova, A., Bebis, G., Pavlidis, I.: Infrared and visible image fusion for face recognition, vol. 5404, pp. 585-596. SPIE (2004)

12. de Diego, I.M., Serrano, Á., Conde, C., Cabello, E.: Face verification with a kernel fusion method. Pattern Recognition Letters (2010)

13. Liu, Z., Tao, Q.: Face recognition using new image representations. In: IEEE INNS - ENNS International Joint Conference on Neural Networks, pp. 1871-1876 (2009)

14. Liu, Z., Liu, C.: A hybrid color and frequency features method for face recognition. IEEE Transactions on Image Processing 17(10), 1975-1980 (2008)

15. Liu, Z., Liu, C.: Robust face recognition using color information. In: Tistarelli, M., Nixon, M.S. (eds.) ICB 2009. LNCS, vol. 5558, pp. 122-131. Springer, Heidelberg (2009)

16. Hwang, W., Park, G., Lee, J., Kee, S.C.: Multiple face model of hybrid fourier feature for large face image set. In: CVPR, pp. 1574-1581 (2006)

17. Su, Y., Shan, S., Chen, X., Gao, W.: Hierarchical ensemble of global and local classifiers for face recognition. In: ICCV, October 2007, pp. 1-8 (2007)

18. Baudat, G., Anouar, F.: Generalized discriminant analysis using a kernel approach. Neural Computation 2(12), 2385-2404 (2000)

19. Cai, D., He, X., Han, J.: Efficient kernel discriminat analysis via spectral regression. In: Proceedings of the International Conference on Data Mining (2007)

20. Stewart, G.W.: Matrix Algorithms Volume I: Basic Decomposition. SIAM, Philadelphia (1998)

21. Zhang, J., Marszałek, M., Lazebnik, S., Schmid, C.: Local features and kernels for classification of texture and object categories: a comprehensive study. International Journal of Computer Vision 73(2), 213-238 (2007)

22. Tan, X., Triggs, B.: Fusing gabor and lbp feature sets for kernel-based face recognition. In: Zhou, S.K., Zhao, W., Tang, X., Gong, S. (eds.) AMFG 2007. LNCS, vol. 4778, pp. 235-249. Springer, Heidelberg (2007)

23. Zhao, J., Wang, H., Ren, H., Kee, S.C.: Lbp discriminant analysis for face verification. In: CVPR, vol. 3, p.167 (2005) 